\title{
Integrating manufacturing database and equipment
}

\author{
Z.K. Lu**, E.H.M. Cheung* \& K.B. Chuah* \\ *Department of Manufacturing Engineering, City University of Hong Kong, \\ HONG KONG Tel:852-7888420; Fax:852-7887660 \\ **Department of Computer Science, Nanjing University of Science \& \\ Technology, CHINA Tel:86-25-432333; Fax:86-25-431622
}

\begin{abstract}
One of the important features of a manufacturing database is that it must be able to communicate effectively with the different elements at different levels of the manufacturing system. For instance, we need to transfer schedule instructions to certain equipment or machines to control the sequence of their operation; download NC programs to appropriate machine tools; capture real-time production data like the status of certain equipment or workin-progress etc. Therefore, in manufacturing database design we need to consider its method of communication with the equipment or other system elements and the related data structure in the database physical model. This paper presents the method of communication between an $\mathrm{Rdb} / \mathrm{VMS}$ manufacturing database and the equipment or other elements in a CIM system via BASEstar and discusses how the data related to the equipment and elements are analysed. It also describes the mechanism for ensuring data integrity in the communication between the database system and equipment or elements of CIM system.
\end{abstract}

\section{Keywords}

Manufacturing database, communication, CIM system, BASEstar

\section{INTRODUCTION}

CIM is essentially about effective communication between the manufacturing database and the different elements or equipment of the manufacturing system it serves. The operation of a manufacturing system requires for example, the issuing of production schedules to shopfloor equipment or machines to control the sequence of their operation; the downloading of NC part programs to appropriate machine tools for the manufacturing of certain parts; the capturing of useful production data such as equipment/machine status or work-in-progress etc. We must organize and manage the data in the manufacturing database in such a way that meets these communication requirements (Chryssolouris, 1992). Thus, early in the manufacturing 
database design stage, we need to consider and decide with care its method of communication with the equipment or other system elements and the related data structure in the database physical model.

VAX/VMS provides the BASEstar software integration platform which can help to bridge the communication gap between equipment and the various application systems of which the manufacturing database system is one. In short, the BASEstar software is designed to facilitate the integration of manufacturing applications and equipment, accelerate the development of applications and device connections, and provide an architecture for the consistent development of manufacturing systems. It also provides the callable services modelled after the VMS system services which provide the building blocks of application and device integration. The manufacturing database can make use of these callable services to define and manipulate data in the BASEstar system to communicate with the various system elements and equipment. The manufacturing database we are talking about here is a relational database system based on the Rdb/VMS. To access the data in this database system, we must use the SQL which provides the programming environment of Rdb. We also need to link this database system with BASEstar via SQL/Services. The SQL/Services then provides application access to Rdb/VMS database from remote computers running a wide variety of operating systems following the client/server model.

\section{COMMUNICATION OF MANUFACTURING DATABASE AND EQUIPMENT}

The key BASEstar component used to integrate manufacturing applications and equipment is its application programming interface (API). The API is a shareable image containing entry points known as callable services. The BASEstar callable services offer developers the ability to:

- Share data among applications

- Control and monitor device operations as well as collect data from plant devices

- Communicate between programs using event-driven messaging

- Control application processing

- Configure some elements of the manufacturing environment

The BASEstar data management callable services provide developers with a common mechanism for defining, organizing, and accessing data in an integrated manufacturing environment. It includes the ability to collect, manage, and distribute current value plant data, automatically notify applications of critical changes in plant information, and export data to an $\mathrm{Rdb} / \mathrm{VMS}$ database for permanent storage.

Based on a CIM environment of VAX/VMS system and application softwares, the proposed manufacturing database is to have a structure as shown Figure 1. There are three levels of data in this database. It is designed to serve the CIM system currently being developed (Cheung et al, $1994_{\mathrm{a} \& \mathrm{~b}}$ ) at the City University of Hong Kong. The first level is the main database which supports all the manufacturing functions of this CIM system. The second level is a host of equipment controller bases which are distributed on the different services to 
control the respective equipment. It is the bridge between the main database and equipment. It serves to accept instructions from the main database, send them to the appropriate equipment, record the status and other information coming from equipment and maintain or update the related data in the main database. The third level provides the equipment interface based on the BASEstar software. The data at this level are used to describe the current status of the manufacturing system; control and monitor the equipment; and control other application processing.

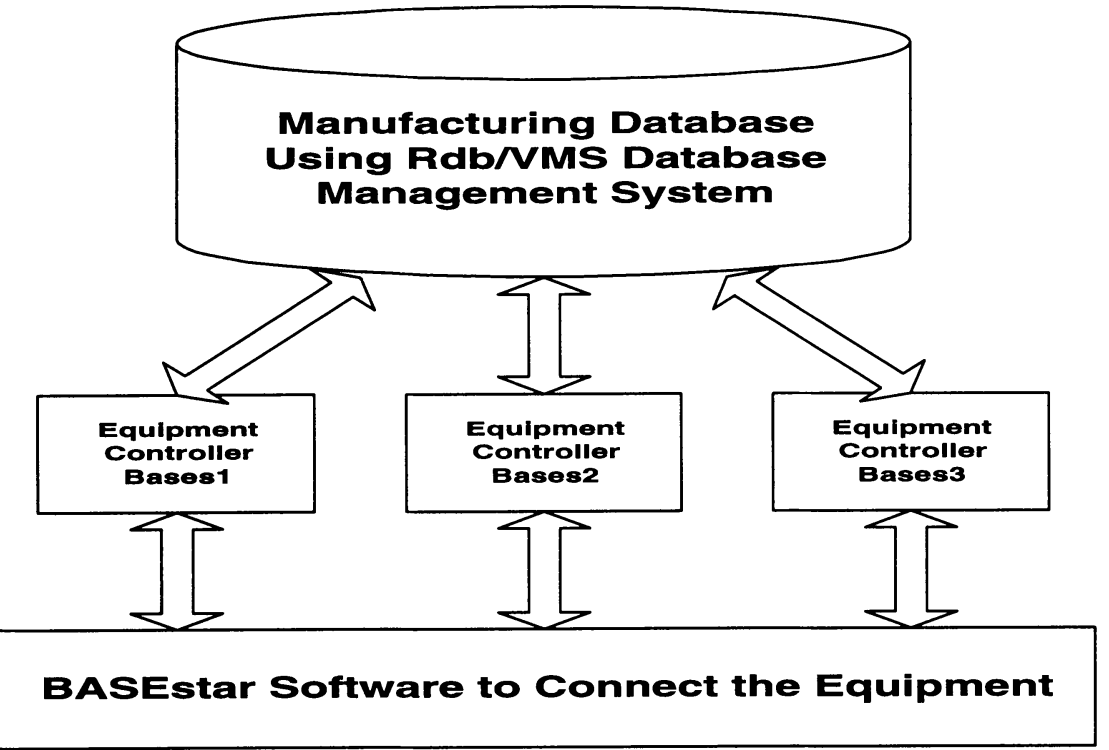

Figure 1 Three-level structure of proposed manufacturing database

\section{DATA ANALYSIS RELATED TO THE EQUIPMENT}

The information required by the management from the manufacturing database would normally be the manufacturing system's main performance indicators like: (1) the status of current jobs; (2) the status of equipment; (3) the setup and processing operation times etc. The computation of these indicators should take into account the requirements or specifications of schedule, quality levels, equipment maintenance, safety, personnel training, and the effects of ongoing efforts such as setup time and operating-time reductions. A distribution of these indicator data among various equipment is to be provided in order to enhance performance. These indicator data are maintained decentrally and the overall data management is performed by a system-wide, global database system.

In the manufacturing database, the data related to the equipment in the shopfloor are such items as: PartL/ULStation, ToolL/ULStation, AGV, Robot, MeasuringEquipment, Operation, MeasuringRecord, AGVSchedule, Equipment, EquipmentStatusReport. In Figure 2, when an instruction in Operation is translated to the equipment, it would effect the relations: 
Equipment, Workpiece, Pallet and FixtureElement. When the status of an equipment is be recorded in the database, it would also effect the relations: Equipment, Workpiece, Pallet, FixtureElement and EquipmentStatusReport. For the same reason, the status of the robot would effect the relations: Robot, Workpiece, Pallet, Fixtureelement, Tool_Using and ToolMagazine. When the AGVSchedule is to be translated to the AGV, it would effect the relations: Workpiece, Warehouse, Buffer, Tool_Using and CentralToolBase. The status of AGV would also effect the Workpiece, Warehouse, Buffer, Tool_Using, CentralToolBase and AGV relations. The result of Measuring Equipment would effect the relations: Workpiece, Tool_using. The status of part load/unload (L/UL) station would effect the Workpiece and Warehouse relations, and the status of tool load/unload (L/UL) station would effect the Tool_Using, ToolMagazine and CentralToolBase relations.

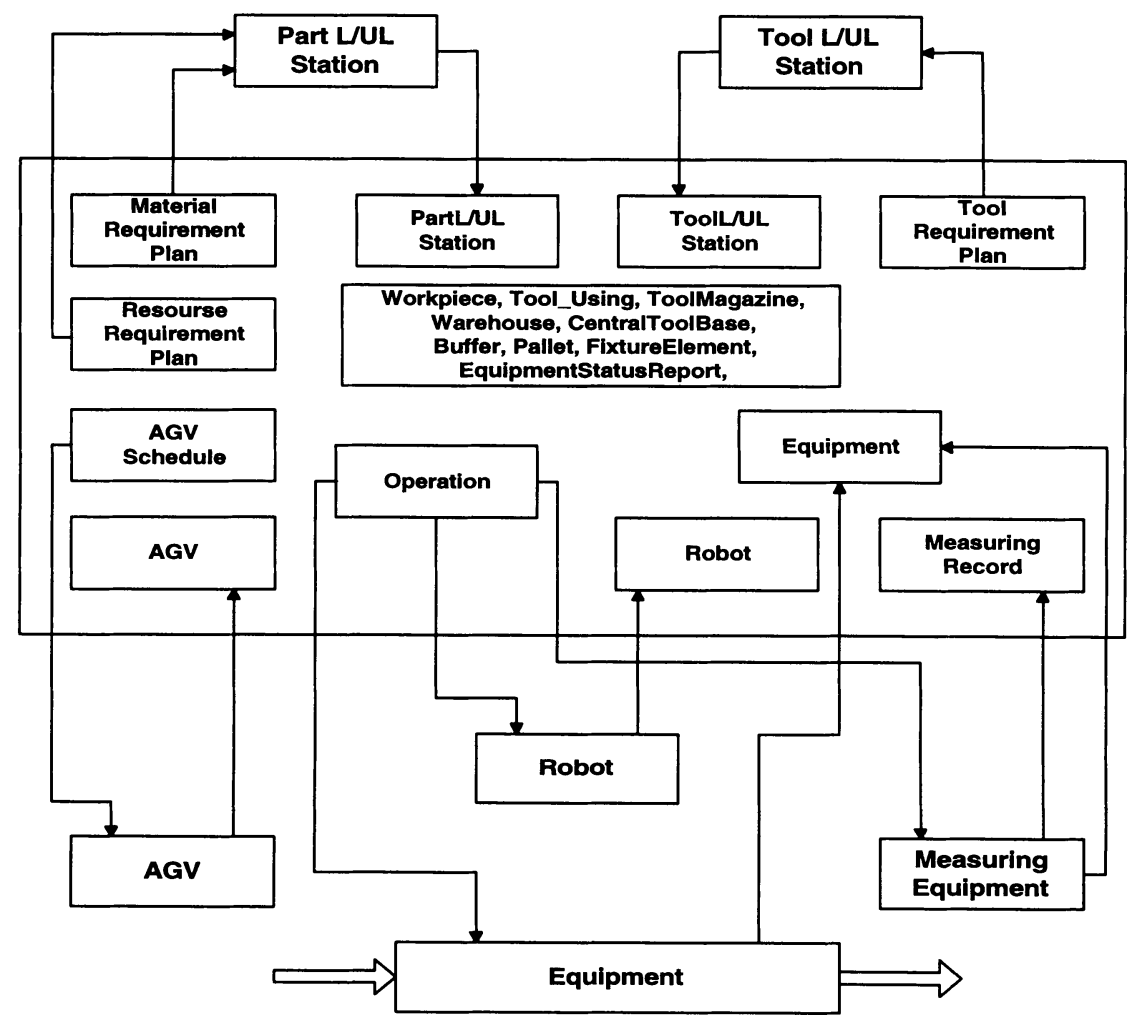

Figure 2 Relationship of data in database and equipment on shopfloor.

Data are defined and referenced in a BASEstar system using logical points. A logical point represents a data item derived from the manufacturing environment. It is stored for immediate 
retrieval in the VAX memory. The value of a logical point can be derived from any of the following sources:

- User input

- Application program

- Mathematical expression

- Plant device

- Point mapping

Users can create logical point definitions and set their value using the command line interface and the menu interface. The BASEstar's data management callable services perform these functions from application programs. In addition to deriving the value of a point from a user or application input, a point value can be the result of a mathematical calculation called expression. Logical points can also be equated to values stored in the memory of a plant device. Device data defined as physical point is collected upon request. Logical points can be connected to physical points to integrate data from plant equipment into the BASEstar application environment.

Once the data is defined as logical and physical points, a BASEstar software can automatically distribute data values when changes occur; equate the value of a logical point to another logical point through point mapping; and export data to other systems such as other databases, applications or computer systems. Examples of these logical points in the manufacturing system are:

- Data related to Operation:

- Data related to Equipment:

- Data related to AGVSchedule:

- Data related to AGV:

- Data related to Robot:

- Data related to MeasuringRecord:

- Data related to MaterialRequirementPlan:

- Data related to ResourceRequirementPlan:

$\begin{array}{ll}\text { instruction } & \operatorname{text}(20) ; \\ \text { status } & \operatorname{text}(1) \\ \text { instruction_no } & \text { text(6) } \\ \text { time } & \text { number(6) } \\ \text { holding_thing } & \text { text(8) } \\ \text { from_buffer_no } & \text { text(4) } \\ \text { to_buffer_no } & \text { text(4) } \\ \text { position_z } & \text { number(4) } \\ \text { direction } & 3 * \text { number(3) } \\ \text { location } & 2 * \text { number(5) } \\ \text { position } & 3 * \text { number(4) } \\ \text { direction } & 3 * \text { number(3) } \\ \text { location } & 2 * \text { number(5) } \\ \text { choices } & \text { text(6) } \\ \text { precision } & \text { text(6) } \\ \text { date } & \text { date } \\ \text { time } & \text { number(6) } \\ \text { material_no } & \text { text(8) } \\ \text { plan_qty } & \text { number(4) } \\ \text { input_buffer_no } & \text { text(4) } \\ \text { date } & \text { date } \\ \text { time } & \text { number(6) }\end{array}$


- Data related to

ToolRequirementPlan:

- Data related to

PartL/ULStation:

- Data related to

ToolL/ULStation: resource_no

plan_qty

input_buffer_no

date

time

tool_no

equipment_no

plan_qty

input_buffer_no

status

send_instruction

finished_instruction

status

send_instruction

finished_instruction $\operatorname{text}(6)$

number(4)

text(4)

date

number(6)

text(6)

text(4)

number(4)

text(4)

text(1)

$\operatorname{text}(6)$

$\operatorname{text}(6)$

text(1)

$\operatorname{text}(6)$

text(6)

Node C

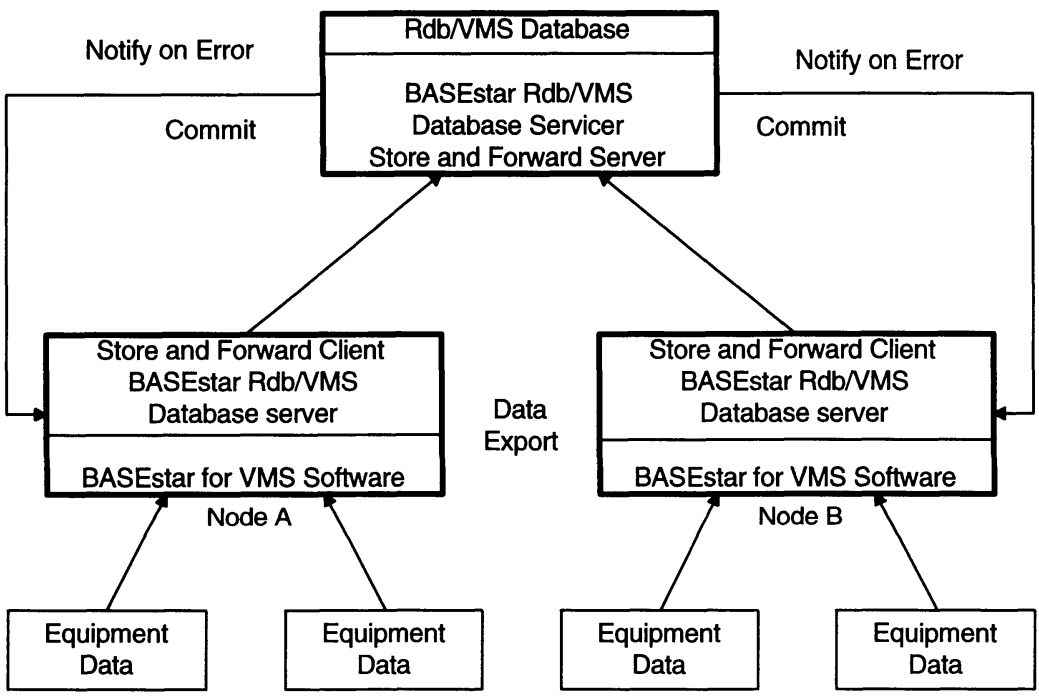

Figure 3 Method of exporting data from equipment.

As mentioned earlier, we can create logical point. We can also connect the logical point to a shopfloor device through BASEstar Device Connect Management (DCM) or to another device connection system. In this case, we must convert the ASCII logical point name into internal binary format which can then be used in any BASEstar Kernel callable services to reduce processing overhead. We can define a logical point as a data structure. This will allow 
efficient processing of complex information by way of adding a data structure definition and then defining the fields within the structure.

Production or shopfloor data collected by BASEstar software can be sent to the manufacturing database. To export data, an external map is created to determine a format for the data. The external map matches the format of BASEstar data that is necessary in the database system. BASEstar data is exported to database using a database server that services as a link between BASEstar software system and the database system as shown in Figure 3. An external map is created so that data elements in a set of logical points can be mapped into a corresponding set of fields in a database table. Each element in an external map can then be added to an external map by the BASEstar callable service.

\section{DATABASE INTEGRITY CONSTRAINTS}

The manufacturing database system described above allows for different users or programs to access the data. The information collected from equipment or other system elements in the manufacturing system can also change the state or content of the database. Therefore, we need to provide a mechanism to maintain the integrity constraints in the manufacturing database system. Because the events which are invoked by the state of the equipment will change the state of manufacturing database, the manufacturing database will automatically maintain the integrity constraints. The events invoked by the equipment in the manufacturing system are listed below:

- Completion of an instruction by robot and other equipment- This event would effect such data as: instruction_no in the data table Equipment; instruction_no, equipment_no, position, status in data table Workpiece; location, position, rotation in data table Pallet; and location, position in data table FixtureElement.

- Completion of a task by AGV- This event would effect such data as: buffer_no in data table Workpiece; storage_status in data table Warehouse; buffer_no in data table Tool_Using; and storage_status in data table CentralToolBase.

- Completion of an instruction by Measuring Equipment- In addition to effecting the data listed in the first event, this event would also effect such data as: weight, volume in data table Workpiece; and volume, tipradcorr, lworkmax, diamaximum in data table Tool_Using.

- Completion of a task by Part L/UL Station- This event would effect such data as: buffer_no in data table Workpiece; and storage_status in data table Warehouse.

- Completion of a task by Tool L/UL Station- This event would effect such data as: buffer_no in data table Tool_Using; and storage_status in data table CentralToolBase.

These events are invoked by different equipment or other system elements which may be located physically at different sites. We must process these events in proper time to maintain 
the states of database and ensure the integrity in the manufacturing database which is concurrently being used by several users or application softwares. To do this, we need to introduce the distributed transaction concept which uses the two-phase commit protocol to ensure the consistency of the database system. This is further elaborated elsewhere (Cheung et al, 1994 a\&c).

\section{CONCLUSION}

This paper reports the result of the final phase of a CIM system database project carried out at the Department of Manufacturing Engineering, City University of Hong Kong. Earlier efforts on the database's conceptual design, logical and physical modelling etc. were presented elsewhere. In this paper, a method of communication between an Rdb/VMS manufacturing database and the equipment or other elements in a CIM system via the BASEstar platform is described. The paper also discusses the data integrity constraints adopted in this database system.

\section{ACKNOWLEDGEMENT}

The authors would like to thank the Croucher Foundation for providing a research grant for this project. They would also like to acknowledge the support provided by the Department of Manufacturing Engineering of City University (formerly City Polytechnic) of Hong Kong and Nanjing University of Science \& Technology.

\section{REFERENCES}

Cheung, E.H.M., Chuah, K.B. and Lu, Z.K. (1994a) Logical Design of A manufacturing Database in CIM Environment, Croucher Foundation Internal Report, Manufacturing Engineering Department, City Polytechnic of Hong Kong, March, 1994

Cheung, E.H.M., Chuah, K.B. and Lu, Z.K. (1994b) The Conceptual Design of a Database System in an Educational CIM Environment, Proc. International Conference on Data and Knowledge Systems for Manufacturing and Engineering, Hong Kong, May 2-4, 1994, $443-$ 448

Cheung, E.H.M., Chuah, K.B. and Lu, Z.K. (1994c) Physical Design of A Manufacturing Database in CIM Environment, Croucher Foundation Internal Report, Manufacturing Engineering Department, City Polytechnic of Hong Kong, June, 1994

Chryssolouris, G. (1992) Manufacturing Systems: Theory and Practice, Springer-Verlag, New York

Steudel, H.J. (1992) Manufacturing in the Nineties, Van Nortrand Reinhold, New York VAX/VMS Workstation series: BASEstar Kernel User's Guide; BASEstar Callable Service Reference; Distributed Transaction in the RDB/VMS. 


\section{BIOGRAPHY}

- Z.K. Lu

Mr Lu graduated with BSc degree in Science in 1982 and later obtained his MSc degree in Computer Science in 1991 from Nanjing University of Science and Technology. He has joined the Department of Computer Science in Nanjing University of Science and Technology since 1982. He worked on a CIM development project funded by the Croucher Foundation in City University of Hong Kong between 1993 and 1994. His current research interests are CIM system analysis, design \& development and Database system design.

\section{- E.H.M. Cheung}

Dr Cheung obtained his MSc in mechanical engineering from University of Manchester Institute of Science and Technology and $\mathrm{PhD}$ from University of Manchester, U.K. His $\mathrm{PhD}$ research was in design of textile machinery. He is a member of the Institution of Mechanical Engineers, a Fellow of the Institution of Electrical Engineers, a Chartered Engineer and a Fellow of the Hong Kong Institution of Engineers. He joined the Department of Manufacturing Engineering, City University (formerly City Polytechnic) of Hong Kong in 1987 when the Department was established and is now the Associate Head of Department. His current interest is CIM system development and implementation, flexible automation and laser material processing.

\section{- K.B. Chuah}

Dr Chuah graduated with a mechanical engineering degree from Loughborough University, U.K. His PhD research was in Shiphull Roughness and Hydrodynamic Drag, a project funded by the US Navy. Since then, he has worked in several U.K. universities as research fellow and lecturer before joining the City University (formerly City Polytechnic) of Hong Kong in 1990. His main research areas are surface metrology, CIM system development \& implementation and project management. 